Research Paper

\title{
Pentraxin 3 overexpression accelerated tumor metastasis and indicated poor prognosis in hepatocellular carcinoma via driving epithelial-mesenchymal transition
}

\author{
Tao Song\#, Cong Wang\#, Cheng Guo, Qingguang Liu, Xin Zheng ${ }^{凶}$ \\ Department of Hepatobiliary Surgery, the First Affiliated Hospital of Xi'an Jiaotong University, Xi'an, Shaanxi 710061, China \\ \#Contributed equally \\ $\square$ Corresponding author: Xin Zheng MD., Ph.D., Department of Hepatobiliary Surgery, The First Affiliated Hospital of Xi'an Jiaotong University, 277 Yanta \\ West Road, 710061 Xi`an, China, E-mail: wyyls@@mail.xjtu.edu.cn \\ (c) Ivyspring International Publisher. This is an open access article distributed under the terms of the Creative Commons Attribution (CC BY-NC) license \\ (https://creativecommons.org/licenses/by-nc/4.0/). See http://ivyspring.com/terms for full terms and conditions.
}

Received: 2018.01.28; Accepted: 2018.05.08; Published: 2018.06.23

\begin{abstract}
As a pattern recognition receptor, pentraxin 3 (PTX3) has been found to exert the pleiotropic roles on a variety of cancers. However, the accurate clinical significance of PTX3 in hepatocellular carcinoma (HCC) has not been well defined. The aim of the present investigation was to determine the expression characteristics, prognostic significance, and the relevant biological effect of PTX 3 in HCC. The expression of PTX3 was evaluated in tumor and adjacent liver tissues from $210 \mathrm{HCC}$ patients using immunohistochemistry staining. And it was found that a marked up-regulation in the expression of PTX3 in the HCC specimens, which was remarkably correlated with high serum AFP level $(P=0.006)$, larger tumor size $(P<0.001)$, liver cirrhosis $(P=0.004)$, advanced TNM stage $(P=0.022)$, PVTT $(P=0.010)$, intra-hepatic metastases $(P=0.019)$, and MVI $(P<0.001)$. PTX3 was identified as an independent predictive factor of poor prognosis by multivariate analysis. Ectopic expression of PTX3 enhanced proliferation, migration, invasion capacities of Huh7 cells and induced EMT phenotype. Silencing PTX3 obtained the opposite results. Moreover, the in vivo experiments confirmed PTX3 induced EMT and promoted proliferation and growth of HCC cells. Collectivelly, these data indicated that PTX3 could accelerate HCC progression through activating EMT and served as a potential predictive factor and therapeutic target for HCC.
\end{abstract}

Key words: PTX3, HCC, EMT, prognosis, predictive factor

\section{Introduction}

Hepatocellular carcinoma (HCC) is the leading type of malignant liver tumor accounting for 70 to $85 \%$ of liver cancer cases worldwide[1] and has been the third most common causes of cancer-related deaths[2]. Liver resection remains the most common one of curative therapies for HCC patients. In spite of great advancement in the therapy regimen for HCC such as anatomical liver resection[3], liver transplantation[4], local and systemic chemotherapy[5], radiofrequency ablation[6], and targeted therapy[7], the post-surgical outcome of HCC patients has hardly improved due to the high rate of tumor recurrence and poor sensitivity to adjuvant chemotherapy drugs[8]. In this regard, it is still urgently needed to uncover the molecular pathways underlying HCC progression and identify the novel biomarkers predicting the post-surgical prognosis of HCC patients.

PTX3, also known as TSG-14, was found in 1992 as the prototypic member of the long-pentraxin subfamily containing NPTX1, NPTX2, PTX4 and the putative integral membrane pentraxin NRP, as well[9-12]. Although there have been several studies reported about the effect of PTX3 on cancer biology, 
the role of PTX3 in cancer remains unclear. Overexpression of PTX3 in tumor tissues was considered as an unfavorable prognostic factor in the setting of pancreatic carcinoma[13], head and neck squamous cell carcinoma[14], gastric cancer[15, 16], breast cancer[17], glioma[18], liposarcoma[19], prostate cancer[20], and lung cancer[21]. On the contrary, PTX3 was found down-regulated and played the ani-tumor actin in melanoma[22] and esophageal carcinoma[23]. Thus, PTX3 seemed to exert a paradoxical function in various cancers.

To our knowledge, it was vague about the correlation between PTX3 expression in tumor tissues and the prognosis of HCCs and its role on HCC progression. In this study, we attempted to figure out the potential of PTX3 to function as a predictive biomarker in HCC after liver resection and provide a better understanding of its bio-function of HCC pathogenesis.

\section{Materials and methods}

\section{HCC patients and samples}

From 2007 to 2009, a total of 210 HCC patients receiving liver resection in Department of Hepatobiliary Surgery, the First Affiliated Hospital of $\mathrm{Xi}^{\prime}$ an Jiaotong University were enrolled in this investigation. All protocols of this study were approved by the ethics committee of the First Affiliated Hospital of Xi'an Jiaotong University according to the Helsinki Declaration of 1964 (No.20070120, 20 Jan 2007). Written informed consent was obtained from all HCC patients enrolled in this study. None of the patients had received systemic chemotherapy or radiotherapy before surgery. All HCC tissues and their corresponding adjacent liver tissues were collected during operation and immediately kept in liquid nitrogen or paraformaldehyde. Follow-up visits were performed for post-surgical survival analysis for up to 96 months after surgery. All clinical features were gained from the medical records. A experienced pathologist provided with the pathological information including tumor diameter, liver cirrhosis, Edmondson-Steiner Classification, and MVI.

\section{Immunohistochemistry (IHC) staining}

IHC assay was carried out on the $4 \mu \mathrm{m}$-thick formalin-fixed, paraffin-embedded section according to the protocal describled previously[24]. The primary antibodies used in IHC staining were from Abcam (MA, USA): rabbit monoclonal anti-PTX3 antibody (Catalog No.: ab125007), mouse monoclonal anti-E-cadherin antibody (ab1416) and mouse monoclonal anti-Vimentin antibody (ab8978). After incubated with the second antibodies, the slides were stained with diaminobenzidine for 3-10 $\mathrm{min}$ at room temperature and then observed by a light microscope.

The expression of target proteins including PTX3, E-cadherin and Vimentin was evaluated independently by two experienced pathologists in the First Affiliated Hospital of Xi'an Jiaotong University. The score of IHC staining was based on the intensity of staining and the proportion of positively stained tumor or liver cells. Staining intensity was classified as follows: 0, negative; 1, weakly positive; 2, moderately positive; 3 , strongly positive. The proportion of positive cells was divided into 5 grades including $0,0-5 \% ; 1,6<25 \% ; 2,26-50 \% ; 3,51-75 \% ; 4, \geq$ $76 \%$. The final IHC score was obtained by multiplying the intensity of staining and proportion of positive cells.

\section{Quantitative reverse transcription-polymerase chain reaction ( $q R T-P C R$ )}

Total RNA of HCC cells was extracted using TRIzol reagent according to the manufacturer's protocol. $1 \mu \mathrm{g}$ of total RNA was used to synthesize cDNA with the help of the PrimeScript RT reagent kit in reverse transcription reactions. qPCR was carried out on an ABI 7300 System using FastStart Universal SYBR Green Master with the following primers: PTX3: Forward 5'-GTAAATGGTGAACTGGCGGC-3', Reverse 5'-ATTCCCCCGGATGTGACAAG-3'; GAPDH: Forward 5'-CGCGCCCCCGG TTTCTA-3', Reverse 5'-GGCTCGGCTGGCGAC-3'. 18s was used as internal control. All experiments were repeated in triplicate.

\section{Western immunoblotting}

The protocal of Western immunoblotting was previously introduced[25]. The rabbit monoclonal anti-PTX3 antibody (Abcam, Catalog No.: ab125007), rabbit polyclonal anti-GLI1 antibody (Abcam, No.: ab49314), rabbit polyclonal anti-SNAIL antibody (Abcam, Catalog No.: ab82846), mouse monoclonal anti-E-cadherin antibody (Abcam, Catalog No.: ab1416) and mouse monoclonal anti-Vimentin antibody (Abcam, No.: ab8978) were used as the primary antibodies, respectively. $\beta$-actin (rabbit polyclonal primary antibody from Abcam, Catalog No.: ab8227) was used as the internal control.

\section{Plasmid Construction and Cell Transfections}

The PTX3 expressing plasimid was built by cloning PTX3 cDNA into pcDNA3.1 vector. Transfection of PTX3 expressing or the vector plasmids into HCC cells was carried out use contg Lipofectamine 2000 transfection reagent (Invitrogen, Carlsbad, USA) according to the manufacturer's protocol. 


\section{Gene knockdown assay}

The siRNA sequences against PTX3 was purchased from ThermoFisher Scientific (Catalog No.: AM16708, USA). And the control siRNA sequences was also from ThermoFisher Scientific (Catalog No.: 4390843). HCC cells were transfected with $30 \mathrm{nM}$ siRNAs with the help pf Lipofectamine 2000 by the user manual. Knockdown of PTX3 was confirmed by both qRT-PCR and Western immunoblotting.

\section{Cell migration and invasion assays}

Scratch wound healing assay was performed in six-well plates to evaluate cell migration. HCC cells were grown to $90 \%$ confluence prior to making an approximately $0.6 \mathrm{~mm}$ wide wound with a pipette tip. Then, the wounds were taken photograph at $0 \mathrm{~h}$ and $36 \mathrm{~h}$ to assess the level of motility. Cell migration ability was quantified by comparing the average migrating distance of HCC cells.

Transwell assays were carried out as before described[26]. Briefly speaking, $5.0 \times 10^{4}$ of HCC cells were planted in DMEM medium without fetal bovine serum (FBS) in the upper chamber of Transwell chamber coated with $40 \mu \mathrm{L}$ Matrigel, while the lower chamber was filled with DMEM medium with 20\% FBS. After 24 hours, HCC cells traversed the filters were fixed with paraformaldehyde, stained with crystal violet after non-invasive cells were scrubbed off the upper surface of chamber. The traversed HCC cells was photographed and counted under a microscopy.

\section{HCC xenograft assay}

All protocolsinvolved in this experiment were approved by the Institutional Animal Care and Use Committee of the First Affiliated Hospital of Xi'an Jiaotong University. Briefly, 8 male nude mice were randomly divided into two groups equally. $1 \times 10^{7}$ Huh7 PTX3 cells were resuspended in $100 \mu \mathrm{l}$ PBS, and injected subcutaneously into the lateral flanks of each nude mouse to be Huh7 PTX3 group. Similarly, $1 \times$ $10^{7}$ Huh7 Vector cells were injected subcutaneouslyin nude mouse to be Huh7 Vector group. HCC xenografts were examined by calipers weakly and the volume of HCC xenografts was calculated using the following equation: Volume $=\mathrm{A} \times \mathrm{B}^{2} \times 0.52(\mathrm{~A}$, length; $B$, width). After 4 weeks, all mice were sacrificed and the xenograft specimens were harvested.

\section{Statistical analysis}

All data were presented as means \pm SD and assessed by Student's t-tests or Mann-Whitney U test for comparison between groups. The clinicopathological features were analyzed by Chi-square tests or Fisher's exact tests between high PTX3 and low PTX3 group. Log-rank test was conducted to compare the
kaplan-Meier survive curves between High and Low PTX3 groups. Statistical analysis was performed using GraphPad Prism 6 or SPSS 18.0. P values less than 0.05 were considered statistically significant.

\section{Results}

\section{Expression of PTX3 was found notably increased in HCC tissues and predicted poor post-surgical survival}

PTX3 expression profiles were evaluated in all of HCC specimens and matched adjacent liver tissues by IHC staining. IHC assay revealed that the PTX3 protein located in cytoplasm predominantly and observed in $192(91.4 \%)$ out of the 210 HCC tissues compared with $136(64.8 \%)$ out of 210 adjacent normal liver tissues (Figure 1A). Based on IHC scoring, it was found that PTX3 expression was up-regulated in HCC tissues in 162 of 210 HCCs $(77.1 \%)$. And Mann-Whitney $U$ test also verified that there was more PTX3 expression in HCC tissues than adjacent liver tissues (Figure 1B). Four pairs of HCC and adjacent liver tissues were randomly selected to assess PTX3 protein expression by Western immunoblotting, which also confirmed that PTX3 expression was aberrantly increased in HCC tissues in contrast to adjacent liver tissues (Figure 1C).

Table 1. Demographic information and clinical characteristics of 210 patients diagnosed with HCC

\begin{tabular}{|c|c|c|c|c|c|}
\hline \multirow{2}{*}{$\begin{array}{l}\text { Clinicopathological } \\
\text { characteristics }\end{array}$} & & \multicolumn{2}{|c|}{ No. of Patients } & \multirow[t]{2}{*}{$X^{2}$} & \multirow[t]{2}{*}{$\mathrm{P}$} \\
\hline & & $\begin{array}{l}\text { Low PDE4a in } \\
\text { HCC }(n=48)\end{array}$ & $\begin{array}{l}\text { High PDE4a in } \\
\text { HCC }(n=162)\end{array}$ & & \\
\hline \multirow[t]{2}{*}{ Age (years) } & $<50$ & 21 & 92 & 2.533 & 0.112 \\
\hline & $\geq 50$ & 27 & 70 & & \\
\hline \multirow[t]{2}{*}{ Gender } & Male & 29 & 108 & 0.638 & 0.425 \\
\hline & Female & 19 & 54 & & \\
\hline \multirow[t]{2}{*}{ HBV infection } & Present & 30 & 135 & 0.410 & 0.522 \\
\hline & Absent & 8 & 27 & & \\
\hline \multirow{2}{*}{$\begin{array}{l}\text { Serum AFP level } \\
\text { (ng/mL) }\end{array}$} & $<400$ & 13 & 18 & 7.507 & $0.006^{*}$ \\
\hline & $\geq 400$ & 35 & 144 & & \\
\hline \multirow{2}{*}{$\begin{array}{l}\text { Tumor diameter } \\
(\mathrm{cm})\end{array}$} & $<5$ & 29 & 27 & 36.24 & $<0.001^{*}$ \\
\hline & $\geq 5$ & 19 & 135 & & \\
\hline \multirow[t]{2}{*}{ Liver cirrhosis } & Present & 42 & 158 & 8.215 & $0.004^{*}$ \\
\hline & Absent & 6 & 4 & & \\
\hline \multirow{2}{*}{$\begin{array}{l}\text { Edmondson-Steiner } \\
\text { Classification }\end{array}$} & $\mathrm{I}+\mathrm{II}$ & 9 & 33 & 0.061 & 0.805 \\
\hline & III + IV & 39 & 129 & & \\
\hline \multirow[t]{2}{*}{ TNM stage } & I + II & 27 & 72 & 5.257 & $0.022^{*}$ \\
\hline & III + IV & 15 & 90 & & \\
\hline \multirow[t]{2}{*}{ PVTT } & Present & 4 & 42 & 6.699 & $0.010^{*}$ \\
\hline & Absent & 44 & 120 & & \\
\hline \multirow{2}{*}{$\begin{array}{l}\text { Intra-hepatic } \\
\text { metastases }\end{array}$} & Present & 2 & 29 & 5.551 & $0.019^{*}$ \\
\hline & Absent & 46 & 133 & & \\
\hline \multirow[t]{2}{*}{ MVI } & Present & 6 & 69 & 14.60 & $<0.001^{*}$ \\
\hline & Absent & 42 & 93 & & \\
\hline
\end{tabular}

Next, we determined the correlation between PTX3 expression in tumor tissues and clinicopathological features of HCC patients. As shown in Table 1, high PTX3 expression in HCC tissues was positively associated with high serum AFP level $(P=0.006)$, larger tumor size $(P<0.001)$, liver cirrhosis $(P=0.004)$, 
A

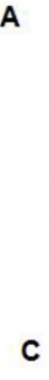

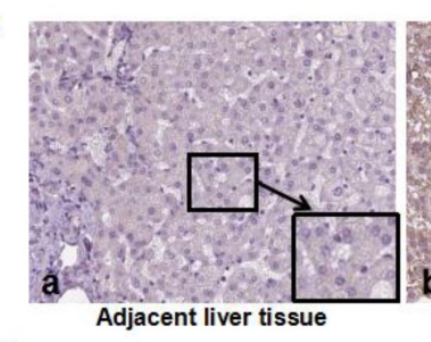

C

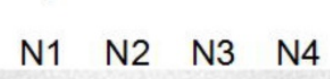

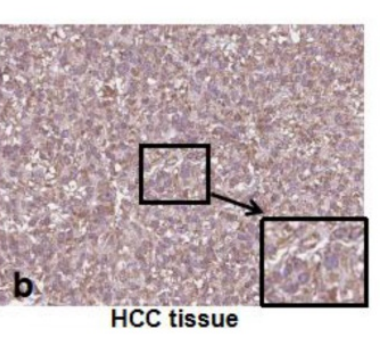
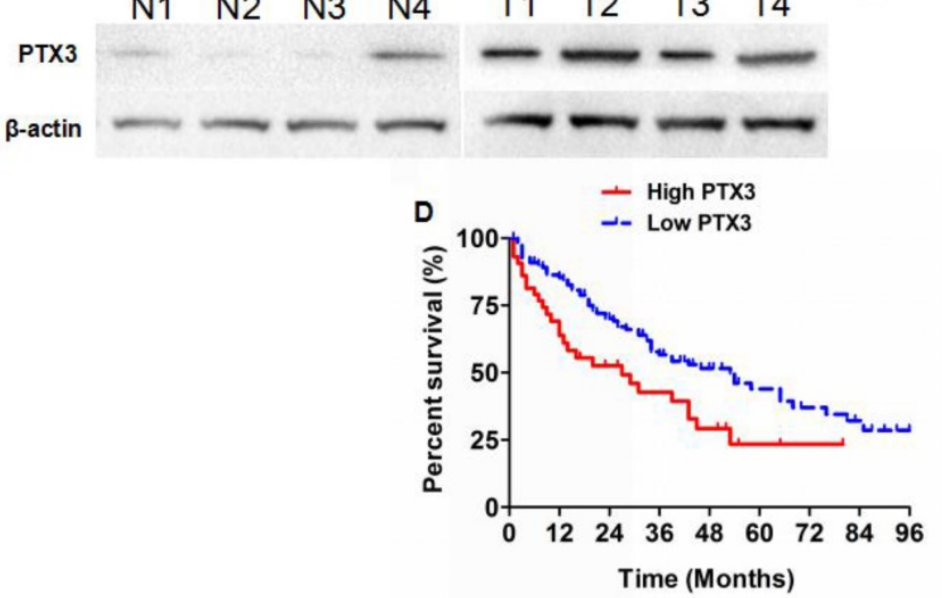

$\mathrm{HR}=2.019,95 \% \mathrm{Cl}(1.183-3.445), \mathrm{P}=0.005$

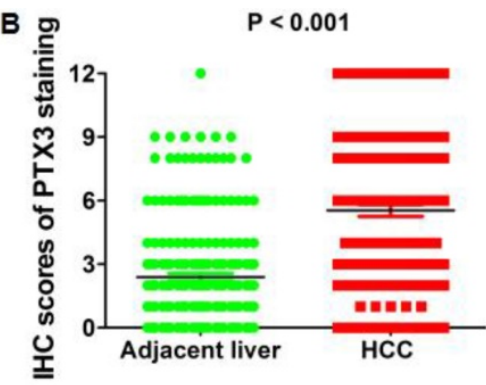

Figure 1. PTX3 was up-regulated in HCC tissues compared with adjacent liver tissues. A IHC staining displayed that there was more positive cells and stronger intensity of PTX3 staining in HCC tissues than adjacent liver tissues (Original magnification $\times 400$ ); B Mann-Whitney U test confirmed that there was more PTX3 expression in HCC tissues than adjacent liver tissues; C PTX3 protein expression was examined in 4 pairs of HCC and matched adjacent liver tissues by Western immunoblotting and it was found that PTX3 expression in HCC tissues was significantly higher than that in adjacent liver tissues; D Comparison of Kaplan-Meier curves revealed that HCC patients with higher PTX3 expression in tumor tissues suffered from notably unfavorable post-surgical prognosis than those with lower PTX3.

advanced TNM stage $(\mathrm{P}=0.022)$, PVTT $(\mathrm{P}=0.010)$, intra-hepatic metastases $(\mathrm{P}=0.019)$, and MVI $(\mathrm{P}$ $<0.001)$, which indicated that up-regulated PTX3 expression in HCC tissues was related with malignant behaviors. We divided 158 HCC patients with the follow-up information into High PTX3 and Low PTX3 group using the ratio of PTX3 expression in HCC/adjacent liver tissuses as cut-off value. Kaplan-Meier curve and the log-rank test were used to determine the role of PTX3 expression on the post-surgical outcome of HCC patients. The median overall survival time of the High and Low PTX3 groups were 27 and 53 months, respectively. The 1, 3 and 5-year survival rates of patients from High PTX3 group were $63.8 \%, 42.7 \%$, and $23.3 \%$, whereas $85.4 \%$, $56.6 \%$, and $39.3 \%$ in Low PTX3 group. Comparision of Kaplan-Meier survival curves between High PTX3 and Low PTX3 group by the log-rank test revealed that HCC patients with higher PTX3 expression in tumor tissues suffered from the unfavorable post-surgical survival than those with lower PTX3 expression $(\mathrm{HR}=2.019,95 \% \mathrm{CI}$ (1.183- 3.445), $\mathrm{P}=$ 0.005, Figure 1D). And both univariate and multivariate analysis were carried out to determine correlations between clinicopathological parameters and postsurgical outcome, which was shown in Table 2. Cox regression model demonstrated that the independent prognostic factors associated with the worse post-surgical survival included high PTX3 expression in HCC tissues, advanced TNM staging, PVTT, intra-hepatic metastases, and MVI. Thus, PTX3 was a potential effective predictive factor for HCC survival.

Table 2. Univariate and multivariate analyses of predictive factors in $\mathrm{HCC}$ patients with liver resection

\begin{tabular}{|c|c|c|c|c|}
\hline \multirow{2}{*}{$\begin{array}{l}\text { Clinicopathological } \\
\text { features }\end{array}$} & \multicolumn{2}{|l|}{ Univariate Analysis } & \multicolumn{2}{|c|}{ Multivariate Analysis } \\
\hline & RR (95\% CI) & P value & RR $(95 \%$ CI $)$ & P value \\
\hline $\begin{array}{l}\text { Age, year }(<50 \\
\text { versus } \geq 50)\end{array}$ & $0.782(0.318-1.726)$ & 0.415 & $0.651(0.277-1.237)$ & 0.524 \\
\hline $\begin{array}{l}\text { Gender (Female } \\
\text { versus Male) }\end{array}$ & $0.354(0.075-1.352)$ & 0.361 & $0.432(0.069-1.686)$ & 0.505 \\
\hline HBV infection & $1.925(0.998-3.399)$ & 0.095 & $1.729(0.895-2.529)$ & 0.122 \\
\hline $\begin{array}{l}\text { Serum AFP level } \\
(\mathrm{ng} / \mathrm{mL})(\geq 400 \\
\text { versus }<400)\end{array}$ & $1.528(0.579-1.981)$ & 0.155 & $1.338(0.578-1.988)$ & 0.257 \\
\hline $\begin{array}{l}\text { Tumor diameter } \\
(\mathrm{cm})(\geq 5 \text { versus }< \\
5)\end{array}$ & $1.752(0.612-2.428)$ & 0.091 & $1.487(0.524-2.197)$ & 0.129 \\
\hline Liver cirrhosis & $2.110(0.977-2.352)$ & 0.124 & $1.857(0.769-3.272)$ & 0.110 \\
\hline $\begin{array}{l}\text { Edmondson-Steiner } \\
\text { Classification (III + } \\
\text { IV versus I + II) }\end{array}$ & $1.381(0.485-1.420)$ & 0.095 & $1.128(0.569-2.155)$ & 0.138 \\
\hline $\begin{array}{l}\text { Advanced TNM } \\
\text { staging }\end{array}$ & $1.932(1.188-4.460)$ & 0.037 & $1.799(1.395-3.715)$ & 0.044 \\
\hline PVTT & $2.695(1.853-5.257)$ & 0.025 & $2.495(1.572-4.913)$ & 0.033 \\
\hline $\begin{array}{l}\text { Intrahepatic } \\
\text { metastases }\end{array}$ & $1.582(1.089-3.228)$ & 0.008 & $1.399(1.090-2.652)$ & 0.019 \\
\hline $\begin{array}{l}\text { Higher PDE4a in } \\
\text { tumor tissue }\end{array}$ & $1.827(1.092-3.057)$ & 0.008 & $1.558(1.164-2.615)$ & 0.012 \\
\hline MVI & $2.358(1.398-5.037)$ & 0.002 & $1.988(1.125-4.289)$ & 0.001 \\
\hline
\end{tabular}




\section{PTX3 promoted proliferation, migration and invasion of HCC cells}

PTX3 expression in LO2 and 5 kinds of HCC cell lines was examined by both qRT-PCR and Western immunoblotting assays. As shown in Figure 2A, it was found that PTX3 expression in normal liver cell LO2 was significantly less than those in all 5 kinds of HCC cell lines. Among HCC cell lines, Huh7 cells had the lowest level of PTX3 expression, while there was more PTX3 expression in MHCC97h cells. Both qRT-PCR and Western immunoblotting assays

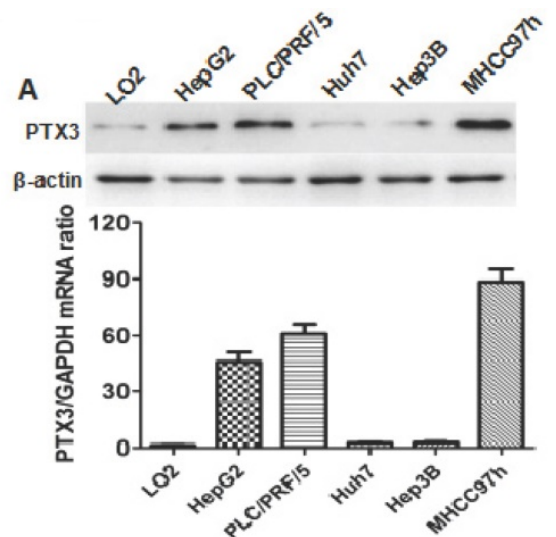

confirmed that transfection of PTX3 expressing plasmid resulted in ectopic expression of PTX3 in Huh7 cells (Figure 2B). BrdU incorporation in Huh7 PTX3 cells was apparently higher than one in Huh7 Vector cells (Figure 2C). Scratching wound healing assay also displayed that migrating distances of Huh7 PTX3 cells were markedly longer than those of Huh7 Vector cells (Figure 2D). Additionally, enforced expression of PTX3 leaded to up-regulation of invasion ability of Huh7 cells notably (Figure 2E).
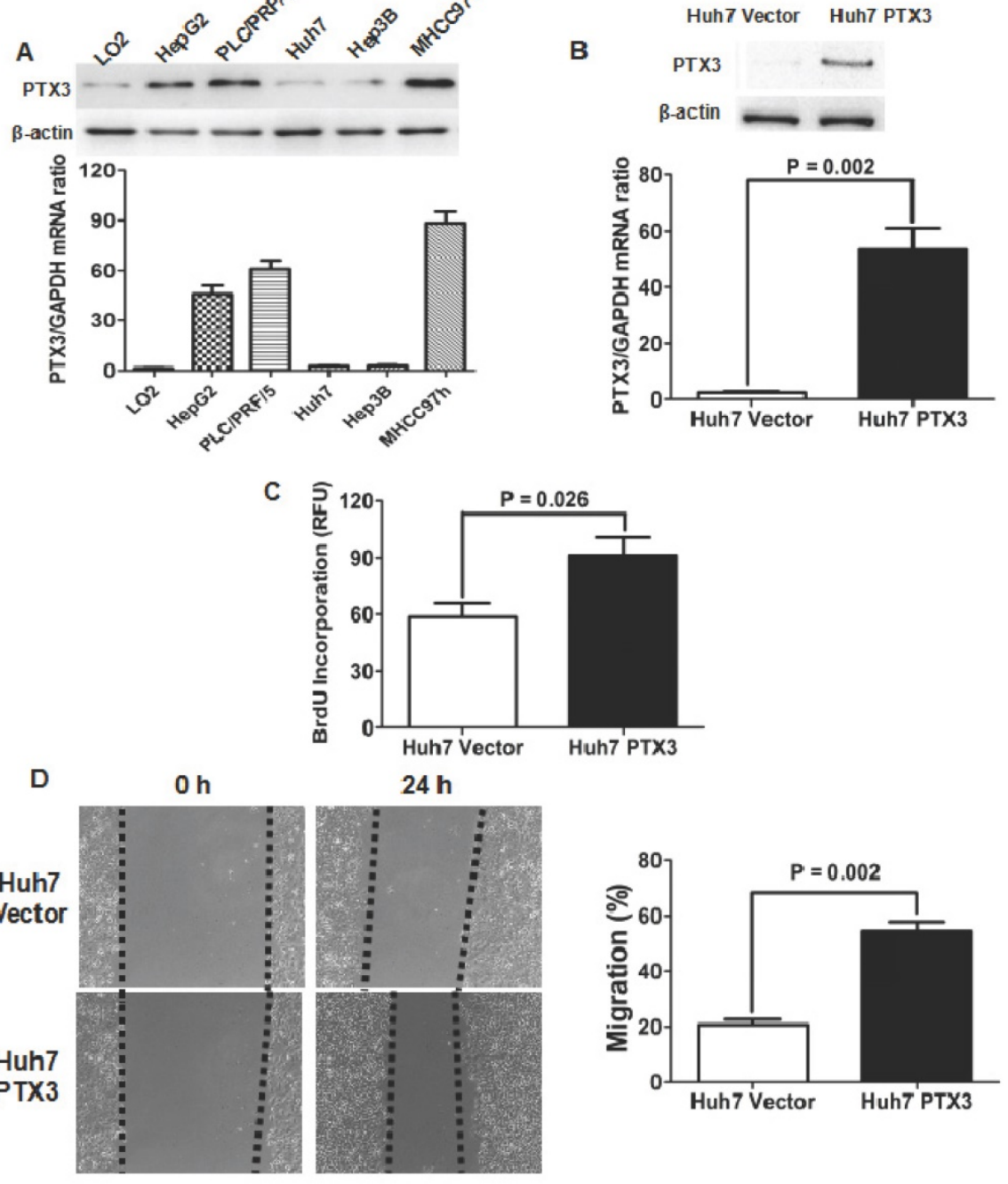

E
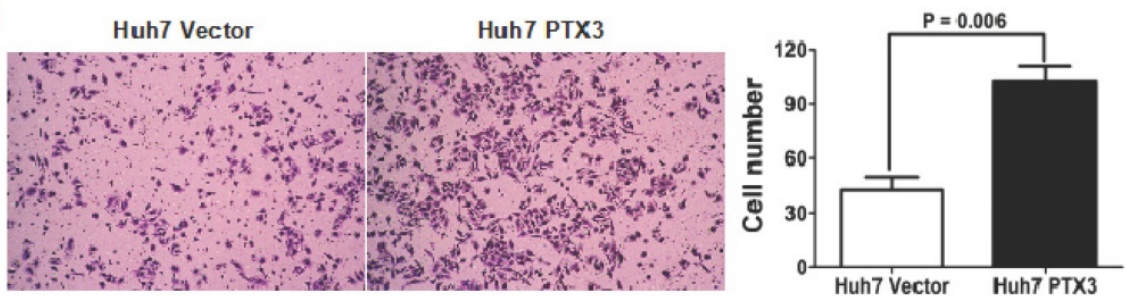

Figure 2. Enforced expression of PTX3 enhanced proliferation, migration and invasion abilities in Huh7 cells. A Both qRT-PCR and Western immunoblotting assays revealed that PTX3 expression in normal hepatocyte LO2 cells was less than 5 kinds of HCC cells; B Transfection of PTX3 expressing plasmid was found to increase PTX 3 expression at the level of mRNA and protein apparently; C ELISA assay showed that enforced expression of PTX3 increased BrdU incorporation of Huh7 cells markedly; D Migration of Huh7 cells was verified to be strengthened magnificently by wound healing assay; E Transwell chamber coated with Matrigel displayed that there were remarkably more Huh7 PTX3 cells penetrating through filters than Huh7 Vector cells. 


\section{Silencing PTX 3 restrained HCC cell proliferation, migration and invasion}

To further figure out the effect of PTX3 on HCC progression, we silenced PTX3 expression in MHCC97h cells expressing the high level of PTX3 using siRNA sequences. As presented in Figure 3A, both qRT-PCR and Western immunoblotting assays showed that PTX3 expression was abated magnificently by transfection of siRNA sequences. Knockdown of PTX3 inhibited proliferation of MHCC97h cells clearly, as assessed by BrdU ELISA assay (Figure 3B). Scratch wound healing assay revealed that migration capacity of $\mathrm{MHCC} 97 \mathrm{~h}$ cells was repressed by silencing PTX3 (Figure 3C). And transwell chamber coated with Matrigel assay verified that invasion capacity of MHCC97h cells was suppressed dramatically by inhibition of PTX3 expression (Figure 3D). In summary, these results supported strongly that PTX3 strengthened HCC cell proliferation, migration and invasion, and consequently promoted HCC progression.

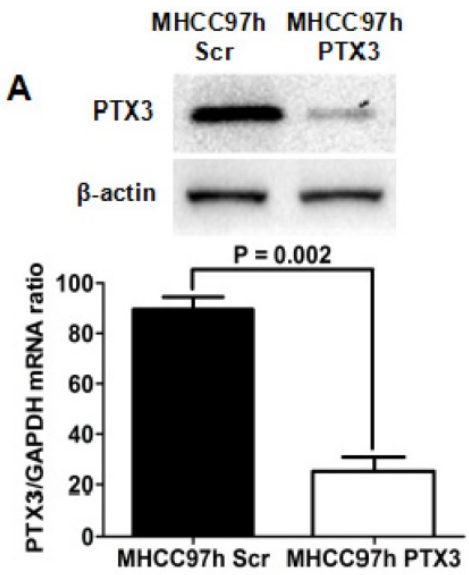

\section{B}
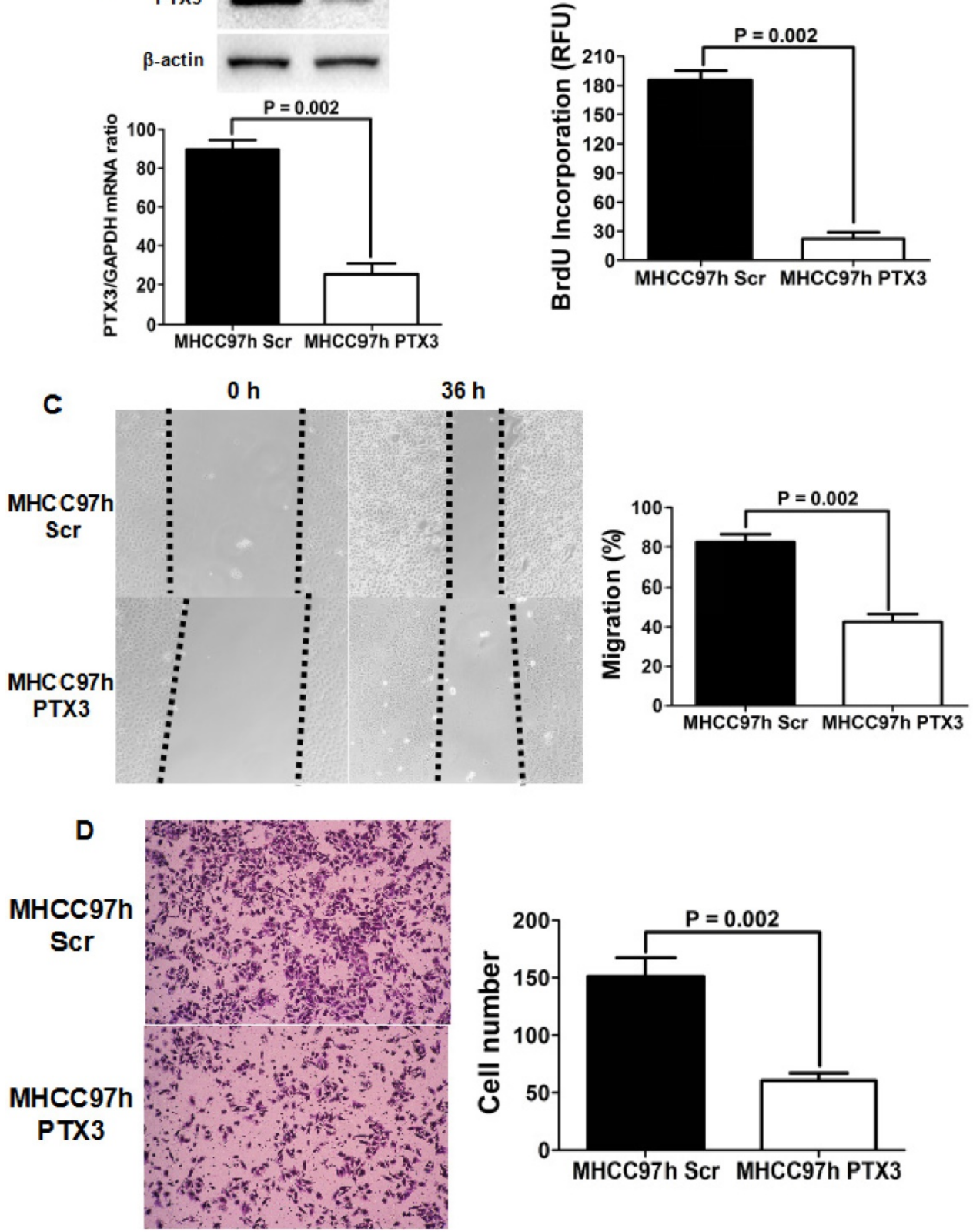

Figure 3. Silencing PTX3 resulted in repression of cell proliferation, migration and invasion in MHCC97h cells. A Both qRT-PCR and Western immunoblotting assays confirmed that PTX3 expression was inhibited by siRNAs transfection; B As measured by ELISA assay, BrdU incorporation was found clearly decreased by knockdown of PTX 3 in MHCC97h cells; C Scratch wounding healing assay showed that migration of MHCC97h cells was attenuated by silencing PTX3 significantly; $\mathbf{D}$ As assessed by Transwell chamber assay, invasion capacity of MHCC97h cells was found repressed by silencing PTX3 dramatically. 


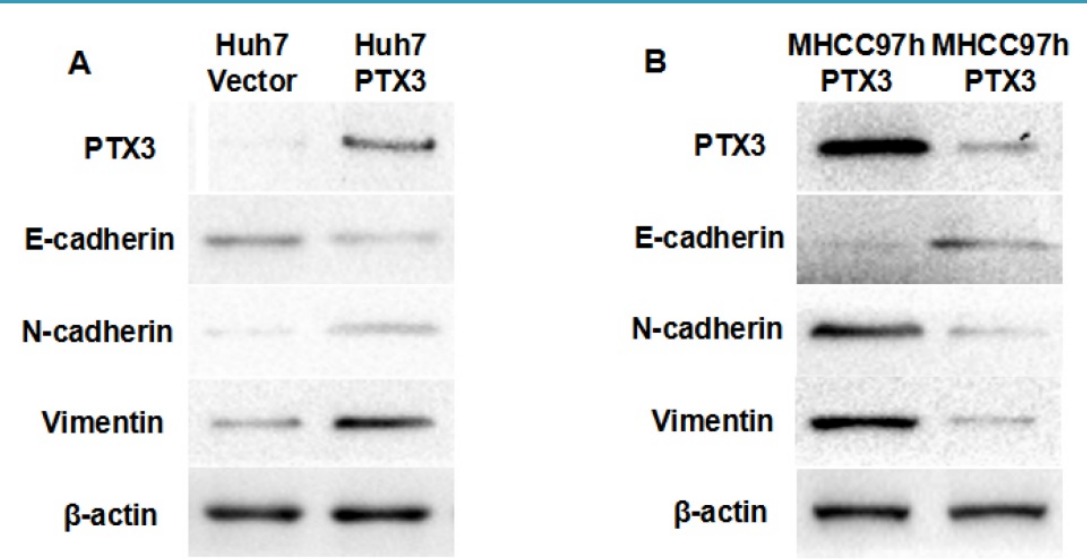

Figure 4. PTX3 induced EMT phenotype of HCC cells. A Western immunoblotting demonstrated that ectopic expression of PTX3 in Huh7 cells increased the expression of $\mathrm{N}$-cadherin and Vimentin, while inhibited E-cadherin expression; B Knockdown of PTX3 was found to resulted in decreased expression of both N-cadherin and Vimentin and up-regulation of E-cadherin by Western immunoblotting assay.

\section{PTX3 enhanced the EMT process of HCC cells}

A growing body of evidences demonstrated that EMT occurred during hepatocarcinogenesis and accelerated tumor progression[26-29]. To determine whether PTX3 accelerated HCC progression through driving EMT, we examined the impact of PTX3 on expression of EMT biomarkers by Western immunoblotting. As shown in Figure 4A, enforced expression of PTX3 in Huh7 cells leaded to loss of E-cadherin which was the well-known epithelial marker, and gain of mesenchymal markers including $\mathrm{N}$-cadherin and Vimentin. Moreover, knockdown of PTX3 increased E-cadherin expression and repressed the expression of both $\mathrm{N}$-cadherin and Vimentin in MHCC97h cells (Figure 4B). To further determine the mechanism that PTX3 drove EMT phenotyle of HCC cells, we examined the expression of well-known EMT inducers. As shown in Figure 4A, ectopic expression of PTX3 increased TWIST1 expression and did not influence the expression of other EMT inducers including TWIST2, SNAI1, ZEB1 and ZEB2.

\section{PTX3 promoted HCC growth in vivo}

To further substantiate the action of PTX3 on tumorigenicity in vivo, we carried out the HCC xenograft experiments on nude mouse model with Huh7 PTX3 and Huh7 Vector cells. As shown in Figure $5 \mathrm{~A}$, the volumes of HCC xenografts in mice inoculated with Huh7 PTX3 cells were remarkably larger compared with controls. The xenografts driven from Huh7 PTX3 cells was found to have more expression of PTX3 and Vimentin, and less E-cadherin expression than those from Huh7 Vector cells (Figure $5 \mathrm{~B})$, as assessed by IHC staining. Moreover, IHC staining of Ki67 revealed that ectopic expression of PTX3 enhanced the growth of HCC cells in vivo (Figure 5C). Collectively, these results indicated that PTX3 played a critical role in HCC cell EMT induction and growth in vivo.

\section{Discussion}

Due to the absence of efficient biomarkers for cancer relapse after curative resection, the post-surgical outcome for HCC patients remains unsatisfied[30, 31]. Amounts of studies revealed that complicated mechanism comprised of distinguishing cancer driver genes and other regulatory factors contributed to HCC pathogenesis and progression[32-35]. PTX3 was also called tumor necrosis factor (TNF)-inducible gene 14 protein (TSG-14) and belonged to the acute-phase protein superfamily which included C-reactive protein (CRP) and serum amyloid P-component (SAP)[36]. Although PTX3 was found to facilitate cancer progression potentially in a variety of cancers including pancreatic carcinoma[13], head and neck squamous cell carcinoma[14], gastric cancer[15, 16], breast cancer[17], glioma[18], liposarcoma[19], prostate cancer[20], and lung cancer[21], limited studies about the role of PTX3 on HCC progression was reported up to now. Cavalcanti et al. examined the PTX3 polymorphisms and its plasma levels in 524 patients with chronic hepatitis $C$ and then found that HCC patients expressed more plasma PTX3 than those with mild or severe fibrosis. And they revealed a remarkable correlation between PTX3 polymorphisms (rs1840680 and rs2305619) and HCC occurrence[37].

In the present study, $77.1 \%$ of HCC tissue samples showed higher expression of PTX3 than the relative adjacent liver tissues and these data indicated that PTX3 was up-regulated frequently in HCC, which was in line with the study from Cavalcanti group. The further analysis showed that over-expression of PTX3 was significantly correlated with malignant bio-behaviors such as high serum AFP level, larger tumor size, liver cirrhosis, advanced TNM stage, PVTT, intra-hepatic metastases, and MVI. The median overall survival time of HCCs expressing 

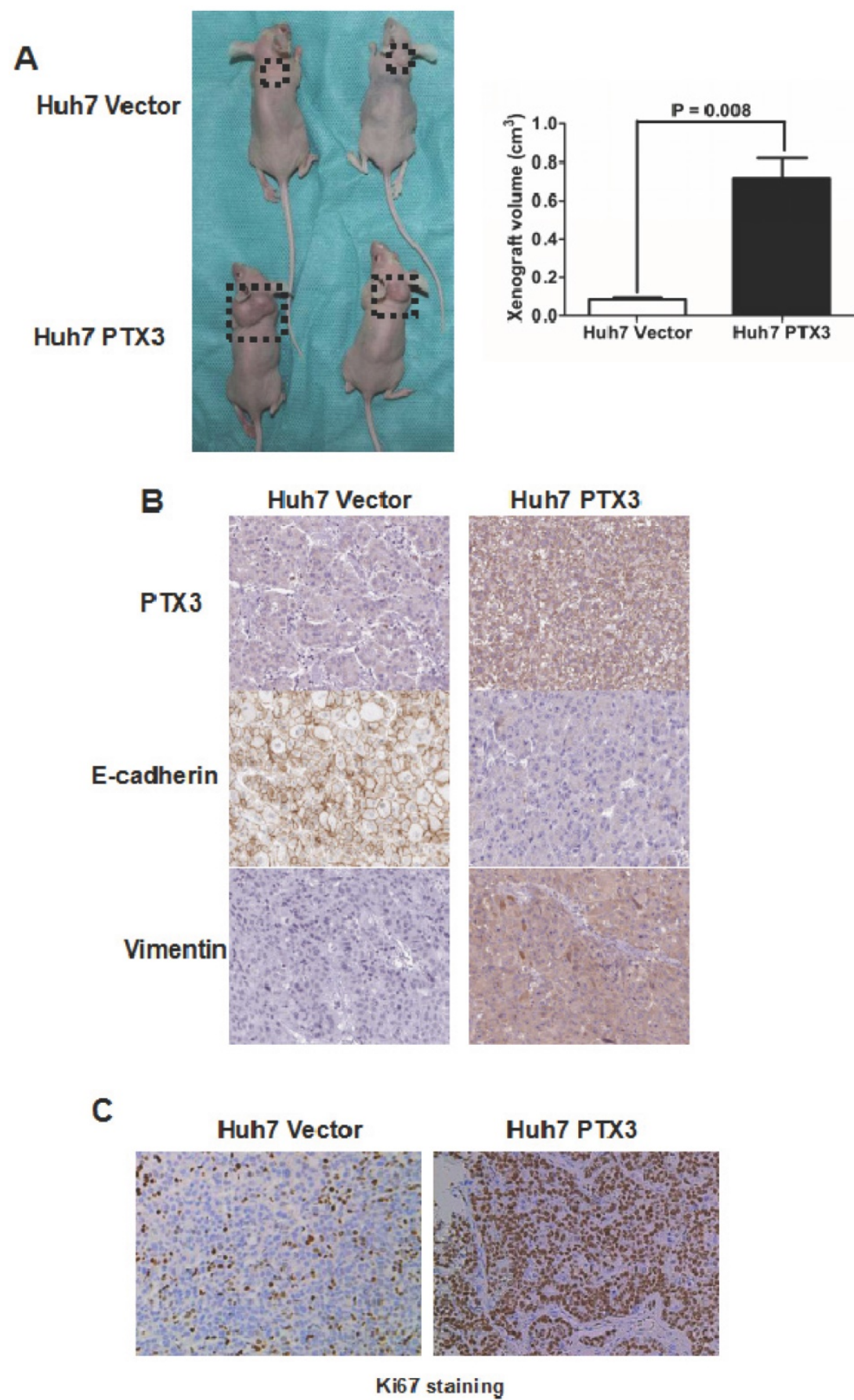

Figure 5. PTX3 promoted Huh7 cells proliferation and growth in vivo. A The volume of HCC xenografts driven from Huh7 PTX3 cells grew larger than those from Huh7 Vector cells; B IHC staining assay showed that HCC xenografts from Huh7 PTX3 cells acquired typical EMT phenotype compared with those from Huh7 Vector cells; C More Ki67 staining Huh7 cells found in HCC xenografts from Huh7 PTX3 cells than those from Huh7 Vector cells by IHC staining assay demonstrated that PTX3 accelerated proliferation of Huh7 cells in vivo.

high level of PTX3 was dramatically shorter than those with low PTX3 expression (27 vs. 53 months). And the 1, 3 and 5-year survival rates of patients with high PTX3 expression was also lower than those of patients with low PTX3 expression. The prognostic significance of PTX3 on HCC patients undergoing liver resection was established by comparison of Kaplan-Meier survival curves. The multivariate analysis identified that PTX3 protein expression level in HCC tissues was the potential independent prognostic factor.

To explore whether PTX3 mediated HCC progression, both in vitro and in vivo experiments were carried out. Ectopic expression of PTX3 enhanced cell proliferation, migration and invasion capacities of Huh7 cells, while increased $\mathrm{N}$-cadherin and Vimentin and repressed E-cadherin. On the other hand, knockdown of PTX3 leaded to the opposite results in the model of MHCC97h cells. Hence, it supported strongly that PTX3 accelerated HCC progression via inducing EMT. The HCC xenograft experiments with nude mouse model confirmed that PTX3 enhanced EMT and accelerated proliferation and growth of HCC cells.

In conclusion, PTX3 played critical roles in HCC cell proliferation, migration and invasion, and induced EMT phenotype of HCC cells. Overexpression of PTX3 in HCC tissues was related with the unfavorable prognosis of HCC patients after surgery. PTX3 has potential to be a promising prognostic factor and therapeutic target for HCC patients receiving hepatectomy.

\section{Abbreviations}

PTX3: Pentraxin 3;

HCC: Hepatocellular carcinoma;

IHC: Immunohistochemistry;

TNM: Tumor-node-metastasis;

EMT: Epithelial-mesenchymal transition;

qRT-PCR: Quantitative reversetranscription-polymerase chain reaction;

PBS: Phosphate Buffered Saline;

FBS: Fetal bovine serum.

\section{Acknowledgements}

This study was supported by grants from National Natural Scientific Foundation of China (81301743 and 81572733 to Xin Zheng), Research Fund for the doctoral Program of High Education of China from Ministry of Education (No. 20120201120090 to Xin Zheng), Key Science and Technology Program of 
Shaanxi Province (No. 2014K11-01-01-21 to Xin Zheng) and the Fundamental Research Funds for the Basic Research Operating expenses Program of Central College sponsored by Xi'an Jiaotong University to Xin Zheng.

\section{Competing Interests}

The authors have declared that no competing interest exists.

\section{References}

1. Venook AP, Papandreou C, Furuse J, de Guevara LL. The incidence and epidemiology of hepatocellular carcinoma: a global and regional perspective. Oncologist. 2010; 15 Suppl 4: 5-13.

2. Siegel RL, Miller KD, Jemal A. Cancer Statistics, 2017. CA Cancer J Clin. 2017; 67: 7-30.

3. Kang KJ, Ahn KS. Anatomical resection of hepatocellular carcinoma: A critical review of the procedure and its benefits on survival. World J Gastroenterol. 2017; 23 : 1139-46.

4. Sapisochin G, Bruix J. Liver transplantation for hepatocellular carcinoma: outcomes and novel surgical approaches. Nat Rev Gastroenterol Hepatol. 2017; $14:$ 203-17.

5. Lencioni R, de Baere T, Soulen MC, Rilling WS, Geschwind JF. Lipiodol transarterial chemoembolization for hepatocellular carcinoma: A systematic review of efficacy and safety data. Hepatology. 2016; 64: 106-16.

6. Nault JC, Sutter O, Nahon P, Ganne-Carrie N, Seror O. Percutaneous treatment of hepatocellular carcinoma: state of the art and innovations. J Hepatol. 2017.

7. Llovet JM, Villanueva A, Lachenmayer A, Finn RS. Advances in targeted therapies for hepatocellular carcinoma in the genomic era. Nat Rev Clin Oncol. 2015; 12: 408-24.

8. Bruix J, Reig M, Sherman M. Evidence-Based Diagnosis, Staging, and Treatment of Patients With Hepatocellular Carcinoma. Gastroenterology. 2016; 150: 835-53.

9. Breviario F, d'Aniello EM, Golay J, Peri G, Bottazzi B, Bairoch A, et al. Interleukin-1-inducible genes in endothelial cells. Cloning of a new gene related to C-reactive protein and serum amyloid P component. J Biol Chem. 1992; 267: 22190-7.

10. Lee GW, Lee TH, Vilcek J. TSG-14, a tumor necrosis factor- and IL-1-inducible protein, is a novel member of the pentaxin family of acute phase proteins. J Immunol. 1993; 150: 1804-12.

11. Garlanda C, Bottazzi B, Bastone A, Mantovani A. Pentraxins at the crossroads between innate immunity, inflammation, matrix deposition, and female fertility. Annu Rev Immunol. 2005; 23: 337-66.

12. Martinez de la Torre Y, Fabbri M, Jaillon S, Bastone A, Nebuloni M, Vecchi A, et al. Evolution of the pentraxin family: the new entry PTX4. J Immunol. 2010; 184: 5055-64.

13. Chorny A, Casas-Recasens S, Sintes J, Shan M, Polentarutti N, Garcia-Escudero R, et al. The soluble pattern recognition receptor PTX3 links humoral innate and adaptive immune responses by helping marginal zone $\mathrm{B}$ cells. J Exp Med. 2016; 213: 2167-85.

14. Chang WC, Wu SL, Huang WC, Hsu JY, Chan SH, Wang JM, et al. PTX3 gene activation in EGF-induced head and neck cancer cell metastasis. Oncotarget. 2015; 6: 7741-57.

15. Choi B, Lee EJ, Park YS, Kim SM, Kim EY, Song Y, et al. Pentraxin-3 Silencing Suppresses Gastric Cancer-related Inflammation by Inhibiting Chemotactic Migration of Macrophages. Anticancer Res. 2015; 35: 2663-8

16. Choi B, Lee EJ, Shin MK, Park YS, Ryu MH, Kim SM, et al. Upregulation of brain-derived neurotrophic factor in advanced gastric cancer contributes to bone metastatic osteolysis by inducing long pentraxin 3. Oncotarget. 2016; 7: 55506-17.

17. Choi $\mathrm{B}$, Lee EJ, Song $\mathrm{DH}$, Yoon SC, Chung $\mathrm{YH}$, Jang $\mathrm{Y}$, et al. Elevated Pentraxin 3 in bone metastatic breast cancer is correlated with osteolytic function. Oncotarget. 2014; 5: 481-92.

18. Locatelli M, Ferrero S, Martinelli Boneschi F, Boiocchi L, Zavanone M, Maria Gaini S, et al. The long pentraxin PTX3 as a correlate of cancer-related inflammation and prognosis of malignancy in gliomas. J Neuroimmunol. 2013; 260: 99-106.

19. Willeke F, Assad A, Findeisen P, Schromm E, Grobholz R, von Gerstenbergk B, et al. Overexpression of a member of the pentraxin family (PTX3) in human soft tissue liposarcoma. Eur J Cancer. 2006; 42: 2639-46.

20. Stallone G, Cormio L, Netti GS, Infante B, Selvaggio O, Fino GD, et al. Pentraxin 3: a novel biomarker for predicting progression from prostatic inflammation to prostate cancer. Cancer Res. 2014; 74: 4230-8.

21. Diamandis EP, Goodglick L, Planque C, Thornquist MD. Pentraxin-3 is a novel biomarker of lung carcinoma. Clin Cancer Res. 2011; 17: 2395-9.

22. Ronca R, Giacomini A, Di Salle E, Coltrini D, Pagano K, Ragona L, et al. Long-Pentraxin 3 Derivative as a Small-Molecule FGF Trap for Cancer Therapy. Cancer Cell. 2015; 28: 225-39.
23. Wang JX, He YL, Zhu ST, Yang S, Zhang ST. Aberrant methylation of the 3q25 tumor suppressor gene PTX3 in human esophageal squamous cell carcinoma. World J Gastroenterol. 2011; 17: 4225-30.

24. Wang $\mathrm{C}$, Yao $\mathrm{B}, \mathrm{Xu} \mathrm{M}$, Zheng $\mathrm{X}$. RIP1 upregulation promoted tumor progression by activating AKT/Bcl-2/BAX signaling and predicted poor postsurgical prognosis in HCC. Tumour Biol. 2016; 37: 15305-13.

25. Zheng X, Xu M, Yao B, Wang C, Jia Y, Liu Q. IL-6/STAT3 axis initiated CAFs via up-regulating TIMP-1 which was attenuated by acetylation of STAT3 induced by PCAF in HCC microenvironment. Cell Signal. 2016; 28: 1314-24.

26. Xu M, Liu Z, Wang C, Yao B, Zheng X. EDG2 enhanced the progression of hepatocellular carcinoma by LPA/PI3K/AKT/ mTOR signaling. Oncotarget. 2017; 8: 66154-68

27. Liu Z, Tu K, Wang Y, Yao B, Li Q, Wang L, et al. Hypoxia Accelerates Aggressiveness of Hepatocellular Carcinoma Cells Involving Oxidative Stress, Epithelial-Mesenchymal Transition and Non-Canonical Hedgehog Signaling. Cell Physiol Biochem. 2017; 44: 1856-68.

28. Gai X, Lu Z, Tu K, Liang Z, Zheng X. Caveolin-1 is up-regulated by GLI1 and contributes to GLI1-driven EMT in hepatocellular carcinoma. PLoS One. 2014; 9: e84551.

29. Zheng X, Vittar NB, Gai X, Fernandez-Barrena MG, Moser CD, Hu C, et al. The transcription factor GLI1 mediates TGFbeta1 driven EMT in hepatocellular carcinoma via a SNAI1-dependent mechanism. PLoS One. 2012; 7: e49581.

30. Finn RS, Zhu AX, Farah W, Almasri J, Zaiem F, Prokop LJ, et al. Therapies for advanced stage hepatocellular carcinoma with macrovascular invasion or metastatic disease: A systematic review and meta-analysis. Hepatology. 2018; 67: 422-35.

31. Giannelli G, Rani B, Dituri F, Cao Y, Palasciano G. Moving towards personalised therapy in patients with hepatocellular carcinoma: the role of the microenvironment. Gut. 2014; 63: 1668-76.

32. Zheng X, Gai X, Ding F, Lu Z, Tu K, Yao Y, et al. Histone acetyltransferase PCAF up-regulated cell apoptosis in hepatocellular carcinoma via acetylating histone $\mathrm{H} 4$ and inactivating AKT signaling. Mol Cancer. 2013; 12: 96.

33. Zheng X, Yao Y, Xu Q, Tu K, Liu Q. Evaluation of glioma-associated oncogene 1 expression and its correlation with the expression of sonic hedgehog, E-cadherin and S100a4 in human hepatocellular carcinoma. Mol Med Rep. 2010; 3: 965-70.

34. Zheng X, Gai X, Wu Z, Liu Q, Yao Y. Metastasin leads to poor prognosis of hepatocellular carcinoma through partly inducing EMT. Oncol Rep. 2013; 29: 1811-8.

35. Zheng X, Gai X, Han S, Moser CD, Hu C, Shire AM, et al. The human sulfatase 2 inhibitor 2,4-disulfonylphenyl-tert-butylnitrone (OKN-007) has an antitumor effect in hepatocellular carcinoma mediated via suppression of TGFB1/SMAD2 and Hedgehog/GLI1 signaling. Genes Chromosomes Cancer. 2013; 52: 225-36

36. Doni A, Garlanda $C$, Mantovani A. Innate immunity, hemostasis and matrix remodeling: PTX3 as a link. Semin Immunol. 2016; 28: 570-7.

37. Carmo RF, Aroucha D, Vasconcelos LR, Pereira LM, Moura P, Cavalcanti MS. Genetic variation in PTX3 and plasma levels associated with hepatocellular carcinoma in patients with HCV. J Viral Hepat. 2016; 23: 116-22. 